\title{
Bücher und Computersoftware
}

A. Engel: Problem-Solving Strategies. 403 Seiten, sFr. 62.-. Springer, Berlin u.a. 1998; ISBN 0-387-98219-1.

Das Buch "Problem-Solving Strategies" ist eine einzigartige Darstellung der wichtigsten Strategien zum Lösen von mathematischen Wettbewerbsproblemen für Gymnasiasten und eine fast unerschöpfliche Sammlung von Aufgaben. Es ist in 14 Kapitel gegliedert, welche jeweils einem Gebiet der Mathematik oder einer Lösungsstrategie gewidmet sind. Es erweist sich als sehr sinnvoll, in einigen Kapiteln einzelne Lösungsstrategien darzustellen, da diese in den verschiedensten Gebieten der Mathematik immer wieder erfolgreich angewendet werden können. Auf diese Weise wird gut sichtbar, welche Aufgabentypen sich mit einer bestimmten Strategie lösen lassen.

Am Anfang jedes Kapitels werden die theoretischen Grundlagen erklärt, sofern sie nicht als allgemein bekannt vorausgesetzt werden können. Diese Grundlagen und die Anwendung der Lösungsstrategien werden anhand von zahlreichen Beispielaufgaben mit ausführlichen Lösungen sorgfältig dargelegt. Dann folgen jeweils viele Aufgaben zum behandelten Gebiet sowie die Lösungen oder zumindest Hinweise und Lösungsskizzen zum allergrössten Teil dieser Aufgaben. Insgesamt enthält das Buch somit eine Sammlung von über 1300 Aufgaben aus verschiedenen Gebieten der Mathematik. Die Probleme stammen unter anderem von über zwanzig der bekanntesten internationalen und nationalen Mathematikwettbewerbe. Das Spektrum reicht von eher einfachen bis zu den schwierigsten je an Wettbewerben gestellten Aufgaben.

Das Buch ist entstanden aus den Vorbereitungen des deutschen Teams auf die Internationale MathematikOlympiade und widerspiegelt die langjährige Erfahrung, die Arthur Engel damit hat. Dies wird unter anderem sichtbar in der Auswahl der Gebiete, welche behandelt werden. So werden neben klassischen Gebieten und Lösungsstrategien wie der euklidschen Geometrie oder der vollständigen Induktion auch Gebiete betrachtet, die über den üblichen Unterrichtsstoff an den Gymnasien hinausführen, wie etwa Zahlentheorie, Funktionalgleichungen oder die Verwendung komplexer Zahlen in der Geometrie. Dagegen fehlen beispielsweise Aufgaben aus dem Bereich der Wahrscheinlichkeitsrechnung oder der Analysis, da diese Gebiete an der Internationalen Mathematik-Olympiade nicht vorkommen.

Das Buch bietet somit einen grossen Vorrat an nicht-routine Aufgaben verschiedener Schwierigkeitsgrade und zahlreiche Anregungen für Lehrer auf der Suche nach Problemen für Wettbewerbe, für MathematikArbeitsgemeinschaften, zur Förderung begabter Schülerinnen und Schüler oder einfach zur Bereicherung des Unterrichts. Insbesondere ist es natürlich hervorragend geeignet zur Vorbereitung auf Wettbewerbe wie die Internationale Mathematik-Olympiade und kann von Schülerinnen und Schülern auch autodidaktisch verwendet werden.

Allen, die sich gerne mit derartigen anspruchsvollen Aufgaben auseinandersetzen möchten, kann ich dieses Buch wärmstens empfehlen.

B. Wollenmann, Rüti

H.R. Schneebeli, H.R. Vollmer: Skalarprodukte - Schwingungen - Signale. 128 Seiten, sFr. 29.80. sabe, Zürich 1998; ISBN 3-252-06054-X.

Hilfreiche Lehrbücher für das neue MAR-Schwerpunktfach "Physik und Anwendungen der Mathematik" (PAM) sind noch rar, und deshalb setzt man sich besonders erwartungsvoll hinter die Lektüre dieses Buches. Um es gleich vorwegzunehmen: Man wird nicht enttäuscht. Die Autoren zeigen, wie man unsere Schüler anhand überzeugender Anwendungen in die Gauss'sche "Methode der kleinsten Quadrate" einführen kann. Im Fischer Lexikon der Mathematik steht sinngemäss, dass "das Gauss'sche Fehlermass eine geometrische 
Betrachtung geradezu aufdrängt”. Diese Auffassung ist das Leitmotiv dieses Buches: Die Gauss-Methode wird ausschliesslich im Rahmen und mit den Mitteln der elementaren analytischen Geometrie behandelt. Der Gewinn ist in zweierlei Hinsicht gewaltig:

- Man sieht erstens in kaum zu überbietender Anschaulichkeit, inwiefern das Gauss'sche Approximationsverfahren optimale Ergebnisse liefert.

- Zweitens kann man auf dem geometrischen Weg Funktionen mit mehreren Variablen und partielle Ableitungen vermeiden.

Die skizzierten Ideen werden bereits im Kap. 1 anhand einfacher Beispiele erprobt. Zuerst werden kurz die erforderlichen Werkzeuge aus der Analytischen Geometrie aufgelistet und dann werden mit der Gauss-Methode die widersprüchlichen Resultate eines physikalischen Versuchs ausgewertet. Im zweiten Beispiel wird "die" optimale Gerade durch drei Punkte berechnet.

Wenn man sich auf die Gauss-Methode konzentrieren will, kann man die Kap. 2 und 3 über Schwingungen und Kreisbewegungen zunächst überschlagen. Im Kap. 4 wird nämlich zunächst das allgemeine lineare Regressionsproblem gelöst. Dabei berechnen die Autoren die gesuchten Koeffizienten aus didaktischer Absicht auf einem eher beschwerlichen Weg über orthonormierte Vektoren, sog. $n$-Listen. (Im VSMP-Bulletin 77/98 findet sich eine bedenkenswerte Alternativlösung von Maxime Zubler.)

Der Höhepunkt des Buches ist zweifelsohne die luzide Darstellung der diskreten Fouriertransformation (DFT). Anhand konkreter Beispiele erfährt man, dass unter gewissen Bedingungen "wenige" Abtastungen eines periodischen Signals genügen, um das vollständige Signal im wesentlichen rekonstruieren zu können. Dabei ergeben sich die gesuchten Koeffizienten der DFT als Skalarprodukte von $n$-Listen.

Das Kap. 5 enthält verschiedenste praktische Anwendungen der DFT. Am überzeugendsten ist die DFT der Vokale einer bestimmten Stimme, insbesondere auch darum, weil sich dieses Beispiel hervorragend für ein gemeinsames Projekt von Physik- und Mathematiklehrer oder für eine Maturarbeit eignet. Im Kap. 6 findet man einen Ausblick auf die kontinuierliche Fouriertransformation (FT). Mit Staunen realisiert der Laie, dass sich die FT als Grenzwert der DFT ergibt. Im Abtasttheorem wird geklärt, unter welchen Bedingungen die "einfache" DFT die gleichen Ergebnisse liefert wie die "komplizierte" FT. Die Wichtigkeit dieser Frage wird durch eine Zusammenstellung möglicher Fehlleistungen des Abtastens sehr gut motiviert. Wahrscheinlich wird jedoch in diesem interessanten Kapitel die obere Grenze der Möglichkeiten eines Gymnasium erreicht.

Das besprochene Werk eignet sich unbedingt für den zukünftigen PAM-Lehrer. Obwohl die didaktischen Stufen unterschiedlich hoch sind, ist es nicht ausgeschlossen, das Buch auch in der Klasse einzusetzen (evtl. nur für einzelne Module). Schliesslich kann es allen Lesern empfohlen werden, die sich noch nie oder schon lange nicht mehr mit Approximationstheorie befasst haben.

Das Buch enthält spärliche Hinweise darauf, dass die DFT z.B. für die digitale Tonaufzeichnung eingesetzt wird. Aber einmal auf den Geschmack gebracht, werden Schüler (und Lehrer) noch weiterfragen und hier (leider) keine detaillierte Antwort finden... Dieser neue Lehrbuchtyp kann noch immer erfunden werden!

O. M. Keiser, Zürich

R. Strebel: Algèbre, géométrie, astronomie. xvi + 517 pages, Editions Universitaires Fribourg Suisse, Fribourg 1997; ISBN 2-8271-0785-6.

Ce fort et beau volume concerne tout enseignant de mathématiques, qu'il soit candidat ou maître chevronné. L'ampleur de cet ouvrage n'est guère excessive quand on constate l'étendue de la matière qu'il traite et la variété des points de vue qu'il développe.

Chacun des trois chapitres - algèbre, géométrie, astronomie - fait d'abord l'objet d'une présentation claire et détaillée, émaillée de remarques didactiques, et faisant la part belle aux motivations inspirées de contextes culturels, aussi bien historiques qu'actuels. Ce développement initial est alors suivi d'une substantielle section modestement intitulée: exercices. Ceux-ci, groupés selon divers thèmes, approfondissent non seulement les connaissances acquises mais élargissent considérablement les perspectives ouvertes dans le chapitre considéré.

Le chapitre consacré à l'algèbre est structuré sur les extensions successives de la notion de nombre: entiers naturels, nombres premiers; entiers, congruences; nombres rationnels; nombres réels. Mais les thèmes d'exercices qui lui sont associés sont très étoffés. Ils comprennent notamment la combinatoire élémentaire; la preuve par récurrence; des exemples d'anneaux et de semi-anneaux: entiers de Gauss, matrices carrées d'ordre 2, anneaux $\mathbb{Z}_{2}$ et $\mathbb{Z}_{3}$, anneaux de fonctions, semi-anneaux définis dans l'ensemble $\mathscr{P}(E)$ des parties de $E$; discernement 
d'erreurs (par exemple, comment $3>2$ peut entraîner - faussement - 1/8 > 1/4); des applications significatives de l'algorithme d'Euclide; anneaux de polynômes à une indéterminée; factorisation des polynômes; le code ISBN; le petit théorème de Fermat; les carrés magiques et les carrés latins.

Le chapitre de géométrie présente deux niveaux de raffinement: intuition géométrique et fondements de la géométrie euclidienne. Ceux qui les ont lus, repenseront avec émotion aux fameux livres de Choquet et de Dieudonné parus en 1964. La présentation de l'auteur est toutefois bien plus proche des préoccupations des enseignants d'aujourd'hui.

La première partie est consacrée à une intéressante et assez généreuse discussion des pavages du plan. L'auteur montre avec élégance comment, à partir d'observations banales bien exploitées, la démarche mathématique peut conduire à des résultats beaucoup moins évidents. Son propos est étayé par des figures très soignées et par une iconographie saisissante.

La seconde partie débute par un survol des géométries mésopotamienne et égyptienne, et surtout par une critique des fameux Eléments d'Euclide. Cela conduit l'auteur à l'introduction de la métrique euclidienne dans le plan et dans l'espace. Les isométries et les similitudes, traitées dans ce cadre, sont discutées tout au long d'une quarantaine de pages, et débouchent sur le calcul matriciel. Un premier appendice passe en revue le traitement réservé aux similitudes dans un certain nombre de manuels scolaires. Un second appendice, beaucoup plus étendu, intitulé modestement complément sur les angles, aborde en détail, tout au long de 65 pages, un sujet qui peut soulever souvent bien des difficultés dans l'enseignement de la géométrie. C'est aussi l'opportunité d'introduire les fonctions trigonométriques; les nombres complexes n'y font malheureusement qu'une trop brève apparition. Un léger regret: ceux-ci ne sont pas exploités à leur juste valeur dans le traitement de nombreuses questions de géométrie, comme l'exposent, par exemple, les livres de Yaglom ou de Hahn.

La section d'exercices propose, tout comme celle suivant le chapitre d'algèbre, d'innombrables thèmes de réflexion: géométrie babylonienne; découpage de pavages, construction de pavages de formes diverses, pavages duaux des pavages semi-réguliers, autour des pavages semi-réguliers, description de pavages à l'aide de la géométrie analytique; la géométrie analytique comme outil de démonstration; construction à l'aide de la règle et du compas; lieux géométriques; espaces vectoriels, applications linéaires et applications affines; groupes; de l'origine de la géométrie analytique; plan métrique du chauffeur de taxi; groupes de symétries d'un ornement du plan; échantillons d'exercices du CO; similitudes du plan euclidien, similitudes du plan du chauffeur de taxi; matrices carrées régulières d'ordre 2 , l'anneau des matrices $M_{n}(K)$.

Le dernier chapitre, consacré à l'astronomie, est conçu comme un complément au développement plus systématique de la géométrie élaboré au chapitre précédent. L'auteur accomplit ce programme avec un bonheur exceptionnel. Sa présentation d'astronomie sphérique, vivante et très originale, est beaucoup plus attrayante que celle que l'on trouve développée dans la majorité des ouvrages consacrés spécifiquement à ce sujet. Pour l'enseignant de mathématiques, l'intérêt est à la fois théorique et pratique. Théorique, parce que l'auteur en profite pour situer le concept de carte (céleste) dans le contexte plus général de la géométrie du $\mathrm{XX}^{\mathrm{e}}$ siècle. Pratique parce que les situations exposées par l'auteur sont autant de thèmes de travaux pratiques - individuels ou en groupe - ou de projets dans le domaine des applications gymnasiales de l'informatique.

On peut ouvrir ce livre n'importe où; on y découvrira toujours quelque chose d'intéressant: une citation curieuse - parfois reproduite dans une autre langue - une référence historique éclairante, une belle figure, une remarque didactique stimulante, une situation motivante, une présentation originale, l'exercice qui manquait, longtemps recherché.

Cet ouvrage remarquable, dont la lecture est très agréable et dont la consultation est aisée, ne devrait manquer dans aucune bibliothèque personnelle; mieux, il devrait trôner sur la table de travail de chaque maître de mathématiques.

P. Bolli, Le Vaud

Références:

- Choquet, G.: L'enseignement de la géométrie. Hermann, Paris 1964.

- Dieudonné, J.: Algèbre linéaire et géométrie élémentaire. Hermann, Paris 1964.

- Hahn, L.: Complex Numbers \& Geometry. The Mathematical Association of America, Washington 1994.

- Yaglom, I.M.: Complex Numbers in Geometry. Academic Press, New York 1968.

Typesetting \& Layout: mathScreen online, CH-4123 Allschwil 